http://jmscr.igmpublication.org/home/ ISSN (e)-2347-176x ISSN (p) 2455-0450

crossref DOI: https://dx.doi.org/10.18535/jmscr/v10i2.01

\title{
Study on the results of sudden cardiac arrest in the emergency department of Al-Bashir Hospital in Jordan
}

\author{
Author \\ Dr Jafar Mohammad Ahmad Yasin*
}

\begin{abstract}
Sudden cardiac arrest (SCA) is a sudden loss of heart function, breathing, and consciousness. This paper aims to study, evaluate and analyze SCA outcomes and how to improve SCA outcomes. Outcomes are better in in-hospital cardiac arrest (IHCA) than in in-hospital cardiac arrest (OHCA). Patients who received prehospital $C P R$ had a higher CPR than patients who did not receive CPR prior to hospital arrival, and treated with vasopressin, epinephrine, and corticosteroids during CPR compared to inhospital cardiac arrest (IHCA). Neurological improvement, and hospital survival compared to Epinephrine Plus. Through the findings, the study recommended improving pre-hospital care that leads to better outcomes than SCA.
\end{abstract}

\section{Introduction}

The heart is the strong muscle that keeps the blood pumped throughout the body, and the heart needs to work well to flow well blood, which is available by coronary arteries, when atherosclerosis lacks blood flow to the heart muscle, or the death of an area of the heart muscle due to clogged blood flow may leads to what is known as cardiac arrest as an unexpected sudden cardiac arrest, so that blood flow to the brain, and other vital organs of the body, stops and is considered dangerous if not treated within minutes: because it will cause death immediately, and there is a difference between stroke and heart attack; Sudden cardiac arrest occurs after recovery from a heart attack or during a heart attack and occurs in people with heart disease, and may occur to those who are healthy and do not suffer from any heart disease and unless rapid intervention is made to treat cardiac arrest with CPR and defibrillation, it leads to death ${ }^{1}$.
It is important to understand that sudden cardiac arrest is different from a heart attack. In the case of a heart attack, blood flow in one of the arteries that supplies blood to the heart suddenly decreases, leading to severe chest pain and other problems. In a few cases, a severe heart attack can lead to SCA, but not always, and there are many other causes of sudden cardiac arrest including genetic and other diseases of the heart muscle ${ }^{2}$. There are no same symptoms or severity of the symptoms in all people with heart attacks. Some people develop mild pain, while others develop more severe pain. Some people don't show symptoms. Others may have a sudden cardiac arrest. However, the more symptoms and indicators you have, the greater your chance of having a heart attack ${ }^{3}$.

Some heart attacks occur suddenly, but many people show warning symptoms and signs of illness hours, days or weeks before a heart attack. The first warning may be repeated chest pain or 
pressure (angina), which occurs due to activity and can be relieved by rest. Angina is caused by a temporary decrease in blood flow to the heart ${ }^{4}$.

The signs of SCA are immediate and radical and include sudden collapse, no pulse, no breathing, accompanied by loss of consciousness.

Sometimes other signs and symptoms appear before sudden SCA. These may include: chest discomfort, shortness of breath, fast heartbeat, palpitations. But SCA are often occurs without warning ${ }^{5}$.

There are also several causes of SCA: coronary artery diseases, which are the main and most common cause of sudden heart death or cardiac arrest, hypertrophic cardiomyopathy, making it difficult for the heart to pump blood, and coronary artery abnormalities: individuals are sometimes born, with heart arteries (coronary arteries) abnormally connected to each other. ${ }^{6}$ It may not provide adequate blood flow to the heart, and wave elongation syndrome: Inherited heart rhythm disorder can quickly cause a heart failure, leading to fainting. Arrhythmias can be life-threatening, including other causes of death from cardiac arrest in young people: unknown congenital heart disease and myocardial abnormalities, as well as myocarditis, which can be caused by viruses and other diseases, But there is also likely to be an indirect impact due to overcrowding in hospital/emergency systems and patients' reluctance to seek timely medical attention due to fear of coming to hospitals ${ }^{7}$.

SCA rates: ${ }^{8}$

1) Percentages of hypertrophic cardiomyopathy (HCM). In this genetic condition, the walls of the heart muscle usually thicken. The enlarged muscles can impair the heart's electrical system, resulting in a fast or irregular heartbeat (arrhythmia), which can lead to sudden death from a heart attack (17\%).

2) Percentages of people with hypertrophic cardiomyopathy, although not usually fatal, is the most common cause of sudden heart-related death in people under 30 years of age. It is the most common cause of sudden death in athletes. Hypertrophic cardiomyopathy often goes unnoticed (27\%).

3) Percentages of patients with abnormal coronary arteries. Sometimes people are born with abnormally connected heart arteries (coronary arteries). Arteries can become compressed during exercise and not provide adequate blood flow to the heart $(37 \%)$

4) Percentages of myocarditis that can be caused by viruses and other diseases. In addition to long QT syndrome, other disorders of the heart's electrical system, such as Brugada syndrome, can cause sudden death (11\%).

5) Percentages as a result of a strong blow to the chest, such as hitting with a hockey stick or hitting another player. A blow to the chest can lead to ventricular fibrillation if the blow comes at the exact wrong time in the heart's electrical circuit, an average of $19 \%$, sometimes due to a traffic accident or sudden fall.

When sudden cardiac arrest occurs, reduced blood flow to the brain causes unconsciousness. If your heartbeat does not return to normal quickly, brain damage occurs and leads to death. Survivors of cardiac arrest may show signs of brain damage and are treated in hospital with vasopressin, which improves outcomes after CPR compared to epinephrine alone. Addition of corticosteroids to vasopressin and epinephrine during therapy with cardiac proteases, and as monotherapy for postresuscitation shock, improves hemodynamics and attenuates the inflammatory response after cardiac arrest. Methylprednisolone, in particular, is thought to enhance the effects of vasopressin and epinephrine.

\section{Materials and Methods}

Community and sample study:

The sample includes patients over the age of 18 years who arrived at the ED with SCA and 
underwent resuscitation in $\mathrm{ED}$ at Al-Bashir Hospital.

The study was conducted on 186 patients, who were resuscitated between 2020 and 2021, with

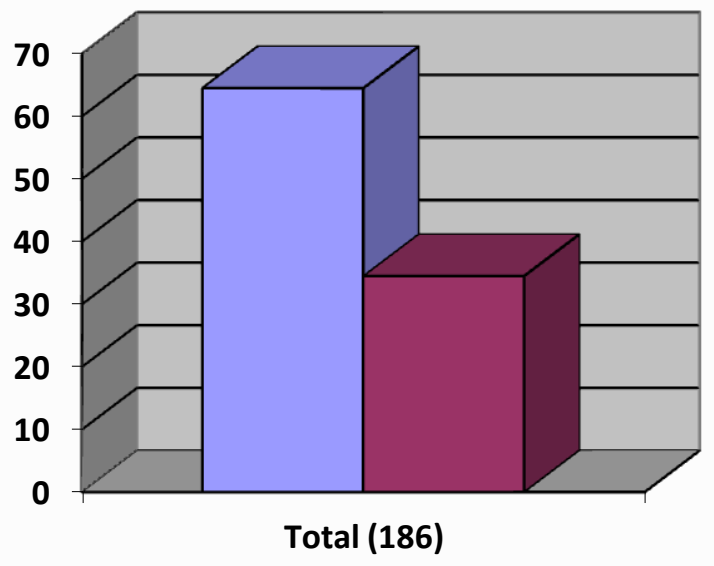

$64,5 \%$ of males and $35,5 \%$ of females, as evidenced by this: $\square$ males (64.5\%)

$\square$ females (34.5)

The number of patients over 50 years of age has reached $87 \%$ and $13 \%$ of patients $<=50$ years, as shown by:

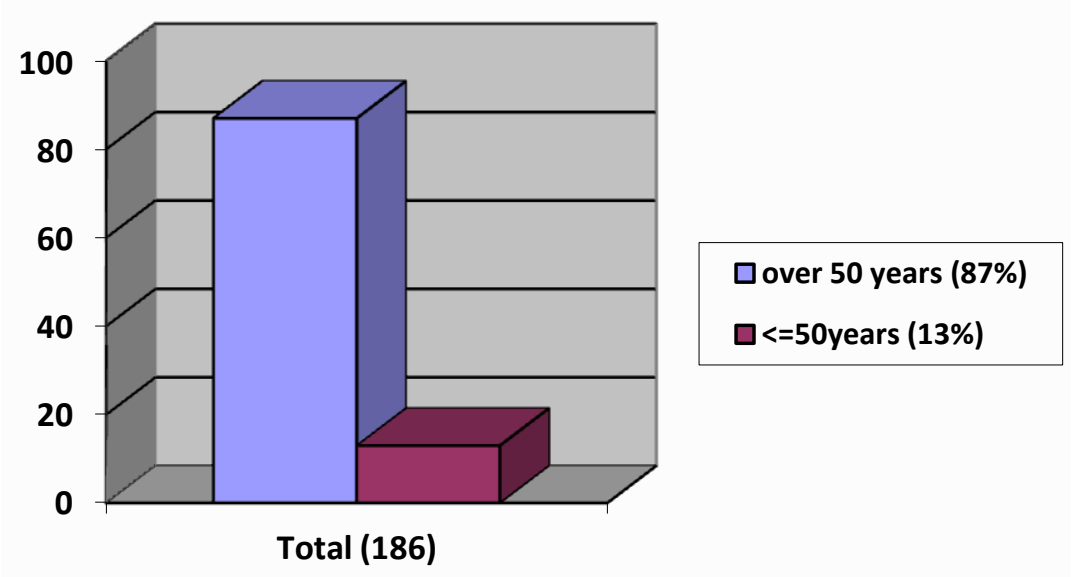

\section{Ethical Issues}

The contact details of each patient and case were examined in complete secrecy, as only their medical condition, gender and age were known. Through the researcher's work as head of the emergency department at Al-Bashir Hospital during the preparation of the research, he reviewed most of the cases that he received and examined by himself, and prepared the results of the study accordingly.

\section{Discussion of the Results}

Total patients (186) pt acording to Sample

IHCA $22,58 \%$ (42) pt

OHCA 77,42\% (144)pt 


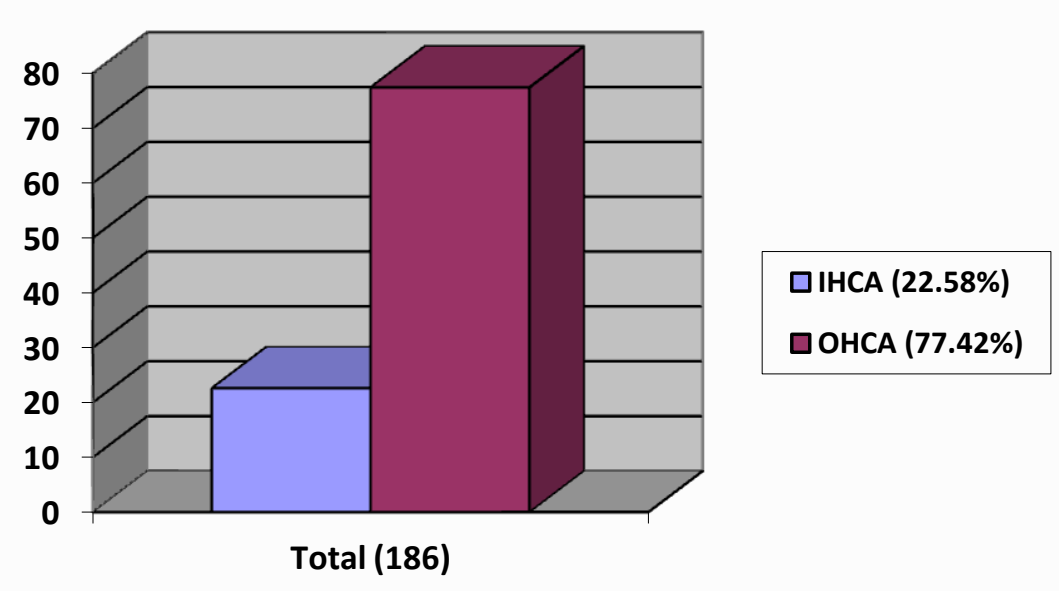

Shockable rhythm $6,5 \%$ (12) pt

Non shockable rhythm 93,5\%(174) pt

shockable rhythm 6.5\% and non shockable rhytm93.5

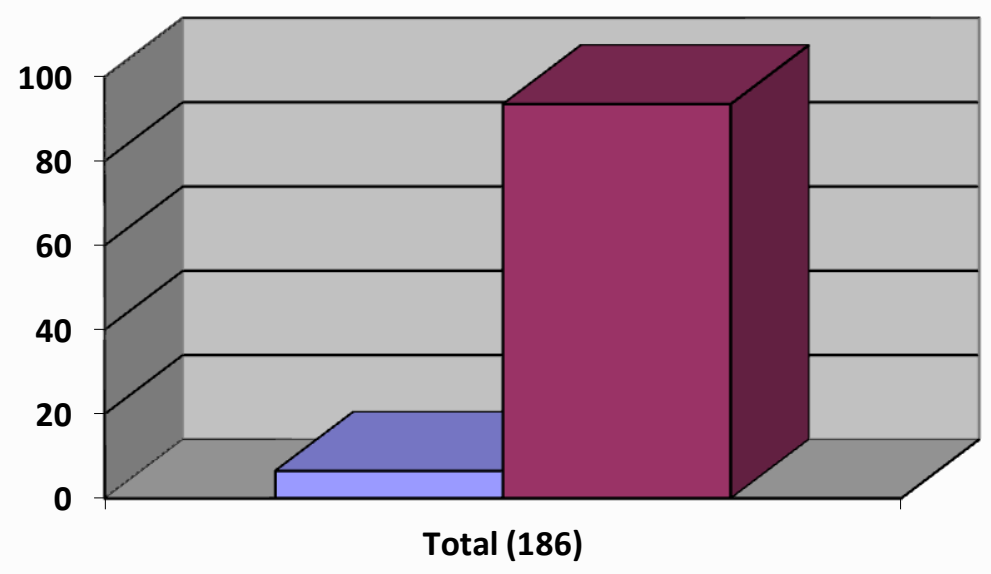

$\square$ Shockable rhythm $6,5 \%(12)$ pt

ROSC overall 15\% (28) pt

ROSC from IHCA 47.6\% (20) pt

ROSC from OHCA $5.55 \%$ (8) pt

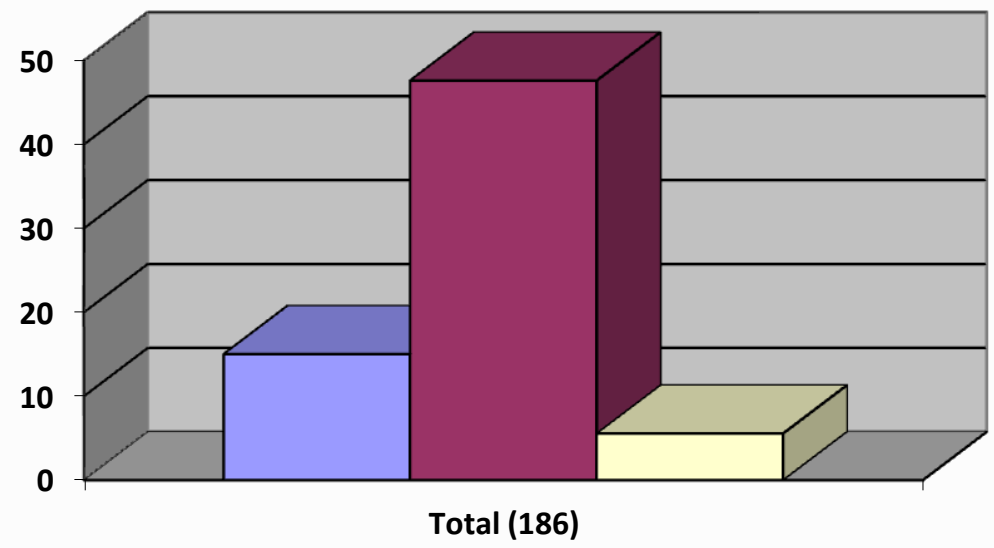

$\square$ ROSC over all 15\% (28) pt

$\square$ ROSC from IHCA $47.6 \%(20)$ pt

口ROSC from OHCA $5.55 \%(8) \mathrm{pt}$
According to IHCA

- $\quad$ shockable rhythm $(23,8 \%) 10$ pt from 42

- $\quad$ non shockable rhythm $(76,2 \%) 32$ pt from 42

- $\quad$ ROSC from all IHCA $(47,6 \%) 20$ pt from 42
- $\quad$ ROSC from shockable rhythm (100\% )10 pt from $10 \mathrm{pt}$

- ROSC from non shockable rhythm( $31 \%$ ) $10 \mathrm{pt}$ from 32 
According to OHCA

- $\quad$ shockable rhythm (1,4\%) 2 pt from 144

- non shockable rhythm (98.6\%)142 pt from 144

- $\quad$ ROSC from all OHCA ( 5.55\%) 8 pt from 144

- ROSC from shockable rhythm (zero\%) zero from $2 \mathrm{pt}$
- $\quad$ ROSC From non shockable rhythm 5,63\% (8Pt from $142 \mathrm{pt}$ )

- All pt with OHCA ROSC received CPR before ED arrival $(20 \%)$ of all OHCA pt received CPR before ED arrival $8 \mathrm{pt}$ from $40 \mathrm{pt}$

\begin{tabular}{|l|c|c|c|c|c|}
\hline & ROSC & $\begin{array}{c}\text { ROSC } \\
\text { shockable } \\
\text { rhythm }\end{array}$ & $\begin{array}{c}\text { ROSC non } \\
\text { shockable } \\
\text { rhythm }\end{array}$ & $\begin{array}{c}\text { shockable } \\
\text { rhythm }\end{array}$ & $\begin{array}{c}\text { non shockable } \\
\text { rhythm }\end{array}$ \\
\hline IHCA & $47.6 \%$ & $100 \%$ & $31 \%$ & $23.8 \%$ & $76.2 \%$ \\
\hline OHCA & $5.55 \%$ & Zero\% & $5.63 \%$ & $1.4 \%$ & $98.6 \%$ \\
\hline
\end{tabular}

According to the results the percentage of ROSC in IHCA is more than OHCA

- All pt with OHCA that become ROSC received CPR before ED arrival but only $(27,77 \%)$ of all OHCA received CPR before ED arrival (40 pt from $144 \mathrm{pt}$ received off before the ER amiral) $20 \%$ of pt that received CPR before ED arrival become ROSC in ED (8pt from $40 \mathrm{pt}$ ) while Zeroy $\%$ of pt with no CPR before $\mathrm{ED}$ arrival become ROSC

Its mean that CPR before ED arrival improves the outcome of SCA, delay in starting CPR after SCA leads to poor results.

The result of this study agreed with a study conducted in a tertiary care center in Greece where a study of 268 consecutive patients applied IHCA to patients under 18 years of age, who had a life expectancy of less than 6 weeks, or cardiac arrest caused by blood Loss: traumatic events (such as rupture of the aorta), and it showed that those who received treatment before arriving at the hospital had a great success rate in cardiac arrest, relieving the pressure of the doctors in the hospital, and the hospital's role is to complete the treatment and provide the necessary care.

\section{Recommendations}

The survival rates from OHCA after CPR still bad we need to improve outcome of SCA by improving prehospital care and decrease risk factor.

\section{References}

1. Gregg C., Fonarow M. and William T. Association between performance measures clinical outcomes for patients hospitalized.JAMANo.297,PP:61-70,2007.

2. Robin, White, Rapid heartbeat, cause and treatment, American Medical Journal, 2020, 1(1), 1014-1120.

3. Gelman E. M., Ehsani A.A. and Campbell M.K. The influence of location and extent of myocardial infarction on long-term ventricular dyshythmia and mortality . Circulation,2019, 60: 805-8141979

4. Halayma, Saad, Myocardial infarction and its effect on coronary artery disease, Radbeck Indian Journal,2020, , 7 (3), 201205 .

5. Health Attack and Angina statistics. American Heart Association.2003,p123.

6. Madeleine, John, Myocardial contractility of athletes, American Journal of Scientific Research 2019, 12(1), 88-92.

7. DeWood M. A., Spores J., Berg G. Acute myocardial infarction . Circulation 68:816. 1983

8. Rich M. Manangement of heart failar in the elderly. Heart fail Rev. No.7, Pp8997,2002.

9. William, Horlick, Sudden Cardiac Arrest Theoretical Study, German Medical Journal,2020, 12(1), 102-109. 